\title{
Die Psyche des Politischen. Was der Charakter über unser politisches Denken und Handeln verrät. Politik und Gesellschaft in der Schweiz
}

\author{
Markus Freitag \\ Zürich 2017: NZZ Libro, 256 S.
}

Sonja Zmerli

Institut d'Études Politiques de Grenoble, Université Grenoble-Alpes E-Mail: sonja.zmerli@iepg.fr
Mit Markus Freitags Schweizer Studie zum Einfluss von Persönlichkeitseigenschaften, den sogenannten Big 5, auf politisches Denken und Handeln erfährt die Politische Psychologie als vergleichsweise junge Teildisziplin der deutschsprachigen Politikwissenschaft eine deutliche Aufwertung. Wer bis dato mit der thematischen Vielfalt der Politischen Psychologie nicht vertraut, aber an einer klugen und kurzweiligen Einführung in einen ihrer vielversprechendsten Forschungszweige interessiert ist, sollte auf die Lektüre dieses Bandes nicht verzichten.

Über 250 anregende Seiten hinweg befasst sich der Autor mit der facettenreichen Forschung zu den Big 5, die konzeptuell in dieser politikwissenschaftlichen Teildisziplin besondere Aufmerksamkeit erfahren und die folgende Persönlichkeitseigenschaften umfassen: Extraversion; Offenheit für Erfahrungen; Gewissenhaftigkeit; Verträglichkeit und Neurotizismus (in Fachartikeln vielfach auch als emotionale Stabilität bezeichnet). Den Leserinnen und Lesern eröffnen sich kenntnisreiche Einblicke in deren wissenschaftliche Ursprünge, politikwissenschaftliche Forschungsbeiträge und internationalen Forschungsstand. Letzterer gibt jedoch nur wenig Auskunft über Schweizer Befindlichkeiten. Zur Behebung dieses offenkundigen Missstands untersucht Freitag einschlägiges Datenmaterial aus vier Schweizer Bevölkerungsumfragen.

Die umfassenden deskriptiven Analysen verstärken manch existierendes Stereotyp, wie etwa, dass Schweizerinnen und Schweizer eher konservativ eingestellt sind. Auch Überraschendes tritt zutage. Bürgerinnen und Bürger des französischsprachigen Landesteils etwa weisen die niedrigsten Verträglichkeitswerte des Landes auf.
Interessierten Leserinnen und Lesern, die bislang noch keine Gelegenheit oder Anlass hatten, sich mit zentralen Fragestellungen der Politischen Psychologie zu befassen, sei insbesondere das einführende Kapitel empfohlen. Pointiert beschreibt und begründet der Autor den Zugewinn, den die deutschsprachige politikwissenschaftliche Forschung durch eine stärkere Berücksichtigung der Persönlichkeitsforschung erfahren würde. Unstrittig ist zwar, dass dieser jüngste Ansatz zur Erforschung politischen Verhaltens in keinem Konkurrenzverhältnis zu etablierten Erklärungsmustern steht, dennoch plädiert Freitag dafür, die Beiträge der Persönlichkeitsforschung als notwendige Ergänzung der konzeptuellen Grundlage zukünftiger empirischer Studien zu nutzen.

Eine Überblicksdarstellung über die psychologischen Ursprünge der Persönlichkeitsforschung und ihre interdisziplinären Entwicklungen bietet das nachfolgende Kapitel, in dem verschiedene Konzepte der Persönlichkeit fundiert und anschaulich vorgestellt und auf ihre Gemeinsamkeiten und Unterschiede hin überprüft werden. Diesen allgemeineren konzeptuellen Erläuterungen schließt sich eine umfassende und auf aktuellen Forschungsarbeiten beruhende Vorstellung der Big 5 an.

Den datenanalytischen Auftakt bildet eine Einführung in die Besonderheiten der Schweizer Persönlichkeitsprofile. Neben den Verteilungen der Big 5 in der Gesamtschweiz erfährt man Wissenswertes über geschlechts-, alters- oder bildungsspezifische Verteilungsunterschiede. Als besonders aufschlussreich erweisen sich die Befunde zu den Stadt-Land-Differenzen oder den Eigenheiten der drei größten Sprachregionen. Die sich anschließenden "Regressions-Stresstests“ attestie- 
ren insbesondere den Indikatoren Geschlecht, Bildung und regionale Herkunft statistische Bedeutung. Insgesamt leisten diese Variablen jedoch nur einen bescheidenen Erklärungsbeitrag zu den Big 5.

Wer sich hingegen für die politischen Dimensionen der Persönlichkeitseigenschaften interessiert, kommt bei der Lektüre der drei nachfolgenden Kapitel auf seine Kosten. Die umfassende Datengrundlage ermöglicht beispielsweise interessante Erkenntnisse zum Zusammenhang zwischen Persönlichkeitseigenschaften und Einstellungen $\mathrm{zu}$ demokratischen Institutionen, politischer Wirksamkeit, Haltungen gegenüber der Politik, politischem Wissen, demokratischen Tugenden, ideologischen Grundhaltungen, Werthaltungen, Einstellungen zur Zusammensetzung des Bundesrates, der Sozial- und Wirtschaftspolitik oder gegenüber Zuwanderung, der Aufnahme von Flüchtlingen und der Europäischen Union. Ein eigenständiges Kapitel ist ferner dem Zusammenhang zwischen Persönlichkeit und Parteipräferenzen gewidmet. Die Analyse der im Zentrum stehenden Indikatoren der Parteiverbundenheit, ihrer Richtung sowie der Parteienwahl offenbaren Erstaunliches: während sich emotional angespannte und extrovertierte Personen mit größerer Wahrscheinlichkeit Parteien zuwenden, sind es die Eigenschaften Offenheit, Gewissenhaftigkeit und Verträglichkeit, die konkretere Aussagen zur Richtung der Parteiverbundenheit und Parteienwahl zulassen. Letzteres gilt insbesondere für die Schweizer Sozialdemokraten und die Schweizerische Volkspartei.

Ergänzt werden die in diesem Band graphisch dargestellten Regressionsergebnisse durch einen umfangreichen und im Internet frei zugänglichen elektronischen Anhang, dem sämtliche Modellbefunde entnommen werden können. Der Band selbst verfügt zudem über einen detaillierten Anhang zur Operationalisierung der Konzepte und aller weiteren genutzten Variablen.

Trotz oder gerade wegen der Fülle des präsentierten Datenmaterials bleiben einige Fragen offen. So sucht man beispielsweise nach einer Begründung für die Wahl der zugrundeliegenden zehnprozentigen Irrtumswahrscheinlichkeit, die für Analysen von Individualdaten seltener herangezogen wird. Vielfach wünscht man sich zudem, dass der Autor mehr Zeit und Raum für die Diskussion der teilweise deutlich voneinander abweichenden Befunde der vier Schweizer Bevölkerungsumfragen aufgewandt hätte. Der Gedanke, dass Weniger möglicherweise Mehr gewesen wäre, drängt sich hier auf.

Den Schlussakkord dieses Bandes lediglich als Fazit zu bezeichnen, erscheint hingegen als Understatement. Sicherlich liegt ein Schwerpunkt auf der Gesamtschau der empirischen Befunde. Bedeutsamer sind hingegen Freitags inspirierende Überlegungen zu den sich eröffnenden Möglichkeiten, aber auch Grenzen der Persön- lichkeitsforschung in der Politikwissenschaft, die sich in Form einer Einladung zur kritischen Auseinandersetzung gleichsam an Fachkolleginnen und -kollegen, Doktorandinnen und Doktoranden sowie Studierende richten.

Mit Die Psyche des Politischen hat Markus Freitag eine eindrucksvolle Studie zum politischen Charakter von Herrn und Frau Schweizer vorgelegt, die sich gewiss als Standardwerk der Persönlichkeitsforschung in der deutschsprachigen politischen Psychologie etablieren wird. 